\title{
Tumour necrosis factor inhibitors slow radiographic progression in patients with ankylosing spondylitis: 18-year real-world evidence
}

\author{
Bon San Koo 지, ${ }^{1}$ Ji Seon Oh, ${ }^{2}$ Seo Young Park, ${ }_{1}^{3}$ Ji Hui Shin, ${ }^{4}$ Ga Young Ahn, ${ }^{5}$ \\ Seunghun Lee, ${ }^{6}$ Kyung Bin Joo, ${ }^{6}$ Tae-Hwan $\mathrm{Kim}^{4}$
}

Handling editor Josef $S$ Smolen

- Additional material is published online only. To view please visit the journal online (http://dx.doi.org/10.1136/ annrheumdis-2019-216741)

For numbered affiliations see end of article.

Correspondence to Dr Tae-Hwan Kim, Department of Rheumatology, Hanyang University Hospital for Rheumatic Diseases, Seoul 04763, Republic of Korea; thkim@hanyang.ac.kr

The results of the study were presented at the American College of Rheumatology Annual Meeting 2019, Atlanta,Georgia (8-13 November).

Received 29 November 2019 Revised 14 May 2020

Accepted 8 June 2020 Published Online First 13 July 2020

Check for updates

(c) Author(s) (or their employer(s)) 2020. No commercial re-use. See rights and permissions. Published by BMJ.

To cite: Koo BS, Oh JS, Park SY, et al. Ann Rheum Dis 2020;79:1327-1332.

\section{ABSTRACT \\ Objectives Tumour necrosis factor inhibitors (TNFis)}

have been suggested to slow radiographic progression in patients with ankylosing spondylitis. However, limitations such as variations in disease activity, complex drug administration and short follow-up duration make it difficult to determine the effect of TNFis on radiographic progression. The aim of the study was to investigate whether long-term treatment with TNFis can reduce radiographic progression in patients with ankylosing spondylitis using 18-year longitudinal real-world data. Methods This retrospective study was conducted between January 2001 and December 2018 at a single centre. Among the 1280 patients whose electronic medical records were reviewed, data of 595 patients exposed to TNFis at least once were included. Among them, time intervals of TNFi exposure or non-exposure were determined in 338 patients ('on the TNFis' or 'off the TNFis' intervals, respectively). The difference in the modified Stoke Ankylosing Spondylitis Spinal Score (mSASSS) change rate between 'on the TNFis' and 'off the TNFis' intervals was investigated.

Results We obtained 2364 intervals of 338 patients (1281 'on the TNFis' and 1083 'off the TNFis' intervals) In the marginal structural model for inverse probability of treatment weighting, the change rate of mSASSS significantly decreased with the use of TNFis ( $\beta=-0.112$, $p=0.004)$, and the adjusted mSASSS changes were 0.848 and 0.960 per year during 'on the TNFis' and 'off the TNFis' intervals, respectively.

Conclusion Compared with treatment without TNFis, treatment with TNFis slowed radiologic progression significantly.

\section{INTRODUCTION}

Ankylosing spondylitis (AS) is a chronic inflammatory disease that causes new bone formation with inflammatory spinal pain. Over the years, ankylosis of the spine progresses, and some patients have disabilities throughout their lives. Several factors are known to affect radiographic progression, but there is a limit to finding factors that can slow down progression. Moreover, determining the effect of a drug for ankylosis is difficult, because it takes a long time to evaluate whether the drug affects the radiographic progression.

Radiographic damage in AS is commonly manifested by erosion and sclerosis beginning early in the corner of vertebra, eventually leading to syndesmophytes and ankylosis. The modified Stoke

\section{Key messages}

What is already known about this subject?

- In some observational cohort study, early and long-term treatment with tumour necrosis factor inhibitors might slow radiographic progression in patients with ankylosing spondylitis. However, it was difficult to evaluate the effect of tumour necrosis factor inhibitors by confounding by indication.

What does this study add?

- Using 18years of electronic medical records and advanced statistical methods, the modified Stoke Ankylosing Spondylitis Spinal Score changes of the intervals exposed and not exposed to tumour necrosis factor inhibitors were significantly different.

How might this impact on clinical practice or future developments?

- By controlling the time-varying confounder, such as the inflammatory markers in real-world data, the amount of reduction in radiographic progression when using tumour necrosis factor inhibitors was presented. Treatment of tumour necrosis factor inhibitor is one of the modifiable factors for radiographic progression.

Ankylosing Spondylitis Spinal Score (mSASSS) is most commonly used to assess radiographic damage. ${ }^{1}$ According to studies measuring mSASSS, a two-unit change per 2 years in mSASSS was considered radiographic progression in $\mathrm{AS}^{2}{ }^{3}$ and various cut-off values are also used to define radiographic progression. ${ }^{4}$ Factors affecting radiographic progression are younger age, male sex, human leucocyte antigen (HLA)-B27 positivity, shorter symptom duration, long disease duration, high disease activity and smoking. ${ }^{2} 3-7$

Recently, tumour necrosis factor inhibitors (TNFis) have been widely used in patients with AS who do not respond to non-steroidal antiinflammatory drugs (NSAIDs). ${ }^{8} 9$ TNFis dramatically improved symptoms by blocking TNF cytokine that plays an important role in inflammation. Although some previous studies showed that they may not have an effect in reducing radiographic progression, ${ }^{10-13}$ several accumulated data on the efficacy of TNFis suggest that TNFis may 
affect radiographic progression in patients with AS. ${ }^{3} 51415$ A high inflammation level at TNFi initiation and long-term TNFi use appear to reduce radiographic progression in a longitudinal cohort study. ${ }^{14-17}$

Real-world evidence refers to clinical evidence obtained from the analysis of real-world data to determine drug effects. ${ }^{18} \mathrm{We}$ expected that with respect to chronic diseases, a study investigating the relationship between drug effects and ankylosis using various healthcare data accumulated over a long period of time, mainly from electronic medical records (EMRs), will provide real-world evidence from patients with AS. ${ }^{19}$ This study aimed to investigate whether long-term treatment with TNFis can reduce radiographic progression in patients with AS using 18-year realworld data.

\section{METHODS}

We retrospectively reviewed the records of 1280 patients between January 2001 and December 2018 at a single centre. All patients were previously diagnosed with AS according to the modified New York criteria. ${ }^{20}$ The Hanyang University Seoul Hospital institutional review board approved this study (HYUH 2014-04-010). Informed consent was waived because this study retrospectively reviewed the EMRs.

Clinical characteristics including age, sex, disease duration from the first to the last follow-up, HLA-B27 positivity, eye involvement with uveitis and peripheral joint involvement with arthritis other than axial joints were investigated. The serum erythrocyte sedimentation rate (ESR) and C-reactive protein (CRP) levels were obtained from EMRs. The Bath Ankylosing Spondylitis Disease Activity Index (BASDAI) ${ }^{21}$ measured approximately twice a year was collected.

\section{Assessment of radiographic progression}

Radiographic images of the cervical and lumbar spine were obtained from patients with AS at an average interval of 2.4 (0.7) years (online supplementary table S1). Two radiologists ( $\mathrm{SL}$ and $\mathrm{KBJ}$ ) independently assessed the images and scored them according to the mSASSS (0-72, (online supplementary methods). ${ }^{1}$ With respect to the accuracy of mSASSS measurement, intraobserver reliability with consistency for a reader was excellent (intraclass coefficient $(\mathrm{ICC})=0.978,95 \%$ CI 0.976 to 0.979). Interobserver reliability with agreement between two readers was also excellent (ICC $=0.946,95 \%$ CI 0.941 to 0.950$)$.

\section{Interval calculation}

Given that we were interested in the period of TNFi administration during this observation period, the time interval was decided based on the TNFi prescription records. The 'on the TNFi' interval is the period from the date the TNFi was prescribed until the 90 days plus $14,3.5,7,60$ and 30 days after the prescription date for adalimumab, etanercept $25 \mathrm{mg}$, etanercept $50 \mathrm{mg}$, infliximab and golimumab, respectively. This rule was made based on the typical dosing interval for each drug and the assumption of the 90-day washout period. If another TNFi prescription was made during the 'on the TNFi' interval; that is, if two 'on the TNFi' intervals overlapped, the two intervals were merged into one. Any time period that the patient was not 'on the TNFi' was considered as the 'off the TNFi' interval. The mSASSS values at the start and end points of the intervals were imputed by the linear interpolation method using the measured mSASSS values before and after each point (figure 1). Data on ESR level, CRP level and BASDAI were also imputed at each time point by linear interpolation. Drug use was simplified as a categorical variable for statistical modelling. If drugs such as conventional diseasemodifying anti-rheumatic drugs (cDMARDs) including sulfasalazine and methotrexate, NSAIDs, and glucocorticoids were prescribed for $>50 \%$ of the entire interval, the drug use interval was considered to be sustained.

\section{Statistical analysis}

All data were summarised as mean (SD) or as percentage. Univariate and multivariable analyses were performed using linear regression with compound symmetry correlation structure for each patient, commonly referred to as generalised estimating equations. The dependent variable was the rate of change in mSASSS (per year), and independent variables included TNFi status (on vs off), levels of ESR and CRP, BASDAI, mSASSS at the start of the interval, concurrent medication status (cDMARDs, NSAIDs and Glucocorticoids) and baseline characteristics, including age, sex and eye and peripheral involvement.

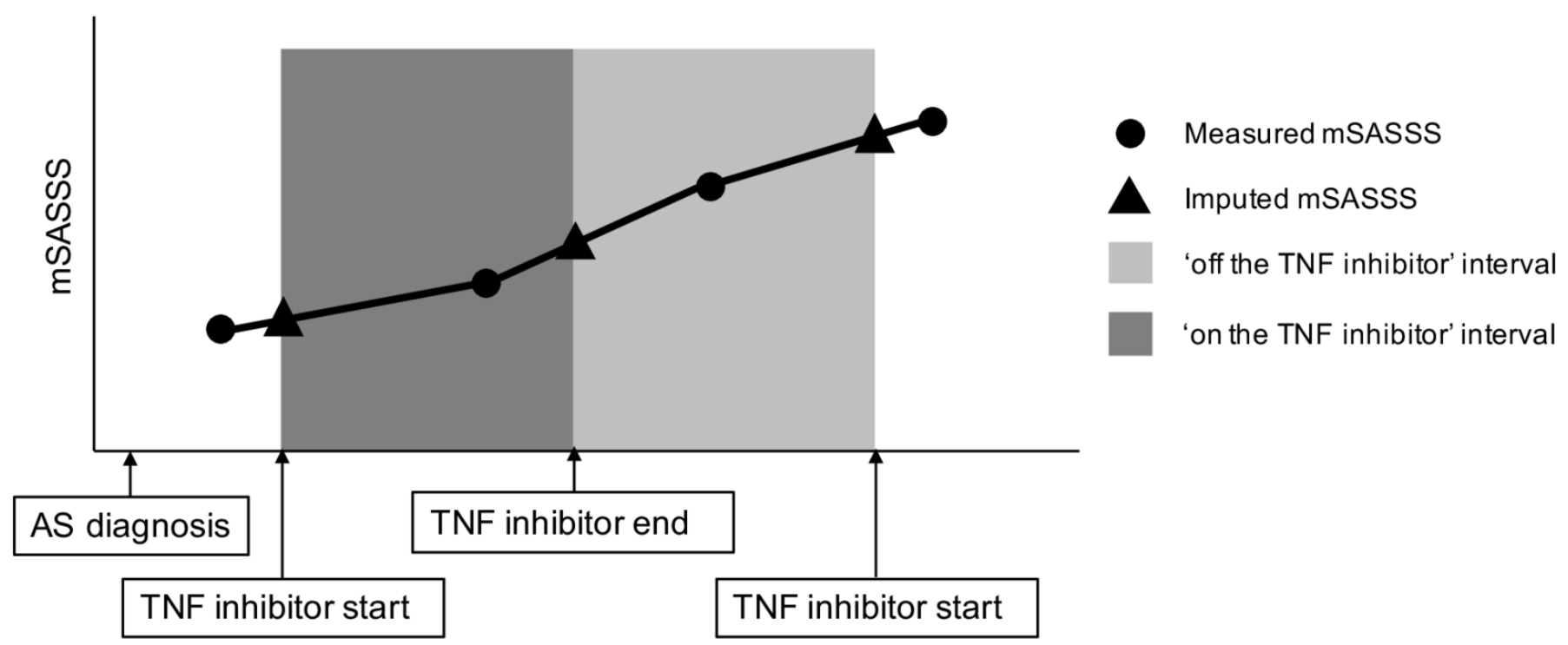

Figure 1 Definition of interval by TNFi use. Imputed mSASSS was obtained by interpolation of the measured mSASSS values before and after the start or discontinuation of the TNFi. AS, ankylosing spondylitis; mSASSS, modified Stoke Ankylosing Spondylitis Spinal Score; TNFi, tumour necrosis factor inhibitor. 
We constructed a multivariable model by removing the variables showing correlation with TNFi use (NSAIDs and CRP and ESR levels), variables with very skewed data (CRP) and variables with too many or too few numbers of positive intervals (HLA-B27 and Glucocorticoids) in the univariate models. Although this kind of multivariable model approach adjusts for the covariates, it has been shown to provide biassed estimate of the treatment effect when there is a time-dependent risk factor that affects the subsequent treatment status and is affected by past treatment history. In this study, time-varying covariates, especially ESR and CRP level, have a potential to work as such risk factors that affect and are affected by treatment status, because physicians tend to prescribe TNFi when the ESR and/or CRP level is high, and TNFi tends to lower the level of these markers. To obtain an unbiased estimate of the TNFi effect, we used a marginal structural model (MSM) with inverse probability of treatment weighting (IPTW), ${ }^{22-24}$ which uses a counterfactual framework to evaluate the causal effect of treatment in the presence of time-dependent confounders that are also intermediate factors. In detail, we calculated the stabilised weight, which has the denominator as the estimated probability of treatment with TNFi based on baseline covariates, ESR (log), CRP (log), BASDAI (square root), mSASSS at the start of the interval, and drugs, including cDMARDs and NSAIDs. The numerator of weight was given by the estimated probability of TNFi treatment based on the baseline covariates only. The actual weight at each time point for each patient was given as the cumulative product of the past weights for that patient. After applying this weight to each time interval, the standardised mean difference between the 'on the TNFi' and 'off the TNFi' intervals became $<0.2$. Based on these weighted data, the change rate of the mSASSS was evaluated by MSM. To estimate CIs and evaluate the significance of TNFis' effect using MSM, we used a sandwich estimator and also performed bootstrapping. P values $<0.05$ were assumed to indicate statistical significance. Finally, we estimated the adjusted mSASSS change rate in each of the 'on the TNFi' and 'off the TNFi' intervals using the change rate of the mSASSS obtained from the multivariable regression model and MSM with IPTW, which was the estimated mSASSS change per year when the other variables were fixed at their mean level. All statistical analyses were performed with R statistical language V.3.5.1.

\section{RESULTS \\ Clinical characteristics of patients and variables related to interval}

Of 1280 patients who were followed for 18 years, 595 were prescribed TNFis at least once (online supplementary figure S1). Among them, 338 patients with at least one 'on the TNFi' and one 'off the TNFi' interval were included. The mean age at diagnosis was 33.1 (9.8) years (table 1). Most patients were male (90\%), and the disease duration was 12.6 (3.6) years (first quantile 10.0 , median 12.5 and third quantile 16.1 years).

Clinical characteristics based on intervals are presented in table 2. We obtained 2364 intervals from 338 patients. Among them, the number of intervals with no actual mSASSS measurements within the interval (completely imputed) was 592 and 758 for 'on the TNFi' and 'off the TNFi' intervals, respectively. The mean duration of the total intervals was 439.1 (646.7) days, whereas that of the 'on the TNFi' and 'off the TNFi' intervals was $633.0(721.0)$ and 209.8 (448.6) days, respectively.

\begin{tabular}{|c|c|c|}
\hline Variables & $\begin{array}{l}\text { No of patients } \\
\text { with data }\end{array}$ & Value \\
\hline Age at diagnosis, mean (SD), year & 338 & $33.1(9.8)$ \\
\hline Female (\%) & 338 & $35(10)$ \\
\hline Disease duration, mean (SD), year & 338 & $12.6(3.6)$ \\
\hline $\begin{array}{l}\text { Duration treated with TNFis per patient, mean } \\
\text { (SD), year }\end{array}$ & 338 & $6.6(3.4)$ \\
\hline $\begin{array}{l}\text { Duration not treated with TNFis per patient, mean } \\
\text { (SD), year }\end{array}$ & 338 & $1.8(2.1)$ \\
\hline HLA-B27 (\%) & 336 & $329(97.9)$ \\
\hline Eye involvement (\%) & 309 & $142(46.0)$ \\
\hline Peripheral joint involvement (\%) & 305 & $170(55.7)$ \\
\hline ESR at baseline, mean (SD), $\mathrm{mm}^{3} /$ hour & 338 & $36.9(32.1)$ \\
\hline CRP at baseline, mean (SD), mg/dL & 338 & $2.6(2.6)$ \\
\hline mSASSS at baseline, mean (SD) & 338 & $17.0(18.6)$ \\
\hline
\end{tabular}

AS, ankylosing spondylitis; BASDAl, Bath Ankylosing Spondylitis Disease Activity Index; CDMARDs, conventional disease-modifying anti-rheumatic drugs; CRP, C-reactive protein; ESR, erythrocyte sedimentation rate; HLA, human leucocyte antigen; mSASSS, modified Stoke Ankylosing Spondylitis Spinal Score; NSAIDs, nonsteroidal anti-inflammatory drugs; TNFis, tumour necrosis factor inhibitors.

\section{Association between mSASSS change rate and covariates in the regression models}

In the univariate model, the mSASSS change rate was significantly associated with age at diagnosis $(\beta=0.017,95 \%$ CI 0.005 to $0.028, p=0.004)$, HLA-B27 positivity $(\beta=0.587,95 \%$ CI 0.050 to $0.123, p=0.032)$ and eye involvement $(\beta=0.432,95 \% \mathrm{CI}$ 0.142 to $0.721, \mathrm{p}=0.003$, table 3 ). Female sex was significantly associated with a decrease in mSASSS change rate $(\beta=-0.415$, $95 \% \mathrm{CI}-0.714$ to $-0.117, \mathrm{p}=0.006)$. Among covariates that measure disease activity, log-transformed ESR at the start of the interval was associated with an increase in mSASSS change rate $(\beta=0.128$, 95\% CI 0.050 to $0.206, p=0.001)$. Among drugs, CDMARDs and TNFis were significantly associated with a decrease in mSASSS change rate $(\beta=-0.335,95 \% \mathrm{CI}-0.538$ to $-0.133, p=0.001$ and $\beta=-0.054,95 \%$ CI -0.099 to -0.009 , $\mathrm{p}=0.017$, respectively).

In the multivariable model, covariates associated with an increase in mSASSS change rate were eye involvement $(\beta=0.420$, 95\% CI 0.124 to $0.717, \mathrm{p}=0.005)$ and ESR at the start of the interval $(\beta=0.199,95 \%$ CI 0.093 to $0.305, \mathrm{p}<0.001)$. Covariates associated with a decrease in mSASSS change rate were female sex $(\beta=-0.561,95 \%$ CI -0.883 to $-0.239, p=0.001)$

Table 2 Summary of variables related to drug use and disease assessment in intervals

\begin{tabular}{lccc}
\hline Continuous variables & Total intervals & $\begin{array}{l}\text { On TNFi } \\
\mathrm{n}=1281\end{array}$ & $\begin{array}{l}\text { Off TNFi } \\
\mathrm{n}=1083\end{array}$ \\
\hline $\begin{array}{l}\text { Duration of intervals, mean (SD), } \\
\text { day }\end{array}$ & $439.1(646.7)$ & $633.0(721.0)$ & $209.8(448.6)$ \\
\hline ESR at start, mean (SD), mm/hour & $20.84(23.75)$ & $23.67(26.37)$ & $17.46(19.66)$ \\
\hline CRP at start, mean (SD), mg/dL & $1.47(1.67)$ & $1.67(1.96)$ & $1.23(1.21)$ \\
\hline BASDAl at start, mean (SD) & $3.52(2.44)$ & $4.00(2.69)$ & $2.95(1.96)$ \\
\hline mSASSS change per year (SD) & $0.85(1.59)$ & $0.82(1.53)$ & $0.89(1.66)$ \\
\hline CDMARDs* $\%)$ & $165(7.0)$ & $103(8.0)$ & $62(5.7)$ \\
\hline NSAIDs* $\%$ ) & $516(21.8)$ & $379(29.6)$ & $137(12.7)$ \\
\hline Glucocorticoids* $\left.{ }^{*} \%\right)$ & $48(2.0)$ & $26(2.0)$ & $22(2.0)$ \\
\hline
\end{tabular}

*Number of intervals prescribed with drugs for $>50 \%$ of the interval period.

AS, ankylosing spondylitis; BASDAI, Bath Ankylosing Spondylitis Disease Activity Index; CDMARDs, conventional disease-modifying anti-rheumatic drugs; CRP, C-reactive protein; ESR, erythrocyte sedimentation rate; mSASSS, modified Stoke Ankylosing Spondylitis Spinal Score; NSAIDs, non-steroidal anti-inflammatory drugs. 
Table 3 Association between mSASSS change rate and covariates

\begin{tabular}{|c|c|c|c|c|c|c|c|c|}
\hline \multirow[b]{3}{*}{ Independent variable } & \multicolumn{4}{|c|}{ Univariate model } & \multicolumn{4}{|c|}{ Multivariable model } \\
\hline & \multirow[b]{2}{*}{ Beta estimate* } & \multicolumn{2}{|l|}{$95 \% \mathrm{Cl}$} & \multirow[b]{2}{*}{$P$ value } & \multirow[b]{2}{*}{ Beta estimate* } & \multicolumn{2}{|l|}{$95 \% \mathrm{Cl}$} & \multirow[b]{2}{*}{$P$ value } \\
\hline & & Lower bound & Upper bound & & & Lower bound & Upper bound & \\
\hline Age at diagnosis & 0.017 & 0.005 & 0.028 & 0.004 & 0.012 & -0.002 & 0.025 & 0.085 \\
\hline Sex (female) & -0.415 & -0.714 & -0.117 & 0.006 & -0.561 & -0.883 & -0.239 & 0.001 \\
\hline HLA-B27 positivity & 0.587 & 0.050 & 1.123 & 0.032 & - & - & - & - \\
\hline Eye involvement & 0.432 & 0.142 & 0.721 & 0.003 & 0.420 & 0.124 & 0.717 & 0.005 \\
\hline Peripheral joint involvement & -0.215 & -0.511 & 0.081 & 0.155 & -0.181 & -0.481 & 0.119 & 0.237 \\
\hline ESR at the start of the interval (log) & 0.128 & 0.050 & 0.206 & 0.001 & 0.199 & 0.093 & 0.305 & $<0.001$ \\
\hline CRP at the start of the interval $(\log )$ & 0.006 & -0.045 & 0.058 & 0.811 & - & - & - & - \\
\hline $\begin{array}{l}\text { BASDAl at the start of the interval (square } \\
\text { root) }\end{array}$ & -0.100 & -0.245 & 0.044 & 0.173 & -0.225 & -0.406 & -0.043 & 0.015 \\
\hline mSASSS at the start of the interval & -0.001 & -0.014 & 0.012 & 0.920 & - & - & - & - \\
\hline cDMARDst & -0.335 & -0.538 & -0.133 & 0.001 & -0.241 & -0.494 & 0.013 & 0.063 \\
\hline NSAIDst & -0.079 & -0.209 & 0.051 & 0.235 & - & - & - & - \\
\hline Glucocorticoidst & 0.134 & -0.283 & 0.552 & 0.529 & - & - & - & - \\
\hline TNFis & -0.054 & -0.099 & -0.009 & 0.017 & -0.054 & -0.101 & -0.008 & 0.022 \\
\hline
\end{tabular}

*Unstandardised regression coefficient.

tNumber of intervals prescribed with drugs for $>50 \%$ of the interval period.

BASDAI, Bath Ankylosing Spondylitis Disease Activity Index; CDMARDs, conventional disease-modifying anti-rheumatic drugs; CRP, C-reactive protein; ESR, erythrocyte sedimentation rate; HLA, human leucocyte antigen; mSASSS, modified Stoke Ankylosing Spondylitis Spinal Score; NSAIDs, non-steroidal anti-inflammatory drugs; TNFis, tumour necrosis factor inhibitors.

and BASDAI at the start of the interval $(\beta=-0.225,95 \% \mathrm{CI}$ -0.406 to $-0.043, p=0.015)$. Among drugs, TNFis were significantly associated with a decrease in mSASSS change rate $(\beta=-0.054,95 \% \mathrm{CI}-0.101$ to $-0.008, \mathrm{p}=0.022)$.

\section{Association between TNFi use and inflammatory markers at the start of the interval}

Inflammatory conditions in patients with AS can affect the choice of TNFi therapy and may even affect the radiographic progression. Considering bias due to inflammatory status, the association between TNFi use and inflammatory markers at the start of the interval was evaluated (online supplementary table S2). The increase in ESR, CRP and BASDAI at the start of the interval had a significant correlation with TNFi use $(\beta=5.946$, $95 \%$ CI 5.012 to $6.881, \mathrm{p}<0.001 ; \beta=0.412,95 \%$ CI 0.339 to $0.484, p<0.001$; and $\beta=0.923,95 \%$ CI 0.808 to 1.039 , $\mathrm{p}<0.001$, respectively). TNFis were significantly prescribed more at the time point with high inflammatory status. Therefore, we confirmed that this tendency in clinical practice may underestimate the effect of TNFis on radiographic progression in conventional regression model.

\section{Causal effect of TNFi on mSASSS change rate}

Considering high inflammatory markers at the start of TNFi therapy, IPTW was used to create a new pseudo-population between the 'on the TNFi' and 'off the TNFi' interval groups (table 4). From the data with IPTW, the mSASSS change rate was compared between 'on the TNFi' and 'off the TNFi' interval groups after adjusting for the effects of time-varying confounders with MSM. The mSASSS change rate significantly decreased when TNFi was used $(\beta=-0.112,95 \%$ CI -0.188 to

Table 4 Original data and data with IPTW

\begin{tabular}{|c|c|c|c|c|c|c|}
\hline \multirow[b]{2}{*}{ Covariates } & \multicolumn{3}{|l|}{ Original data } & \multicolumn{3}{|c|}{ Data with IPTW } \\
\hline & Off the TNFis & On the TNFis & SMD & Off the TNFis & On the TNFis & SMD \\
\hline Age, mean (SD), year & $32.66(8.80)$ & $32.74(8.80)$ & 0.009 & $33.25(9.23)$ & $33.03(8.97)$ & 0.024 \\
\hline Female (\%) & $101(10.6)$ & $120(10.7)$ & 0.004 & $88.2(10.5)$ & $107.0(10.8)$ & 0.011 \\
\hline Eye involvement (\%) & $468(49.0)$ & $549(48.8)$ & 0.002 & $447.6(53.2)$ & $506.7(51.3)$ & 0.038 \\
\hline Peripheral joint involvement (\%) & $540(56.5)$ & $630(56.0)$ & 0.009 & $511.4(60.8)$ & $583.5(59.1)$ & 0.035 \\
\hline ESR at the start of the interval, mean (SD), log & $2.30(1.07)$ & $2.52(1.17)$ & 0.190 & $2.42(1.14)$ & $2.51(1.19)$ & 0.071 \\
\hline CRP at the start of the interval, mean (SD), log & $0.02(0.47)$ & $0.17(0.64)$ & 0.276 & $0.14(0.62)$ & $0.19(0.66)$ & 0.083 \\
\hline $\begin{array}{l}\text { BASDAI at the start of the interval, mean (SD), } \\
\text { square root }\end{array}$ & $1.60(0.57)$ & $1.84(0.72)$ & 0.365 & $1.72(0.63)$ & $1.82(0.74)$ & 0.144 \\
\hline mSASSS at the start of the interval, mean (SD) & $20.00(20.07)$ & $19.43(19.96)$ & 0.028 & $20.98(20.14)$ & $20.17(20.32)$ & 0.040 \\
\hline cDMARDs $*(\%)$ & $27(2.8)$ & $56(5.0)$ & 0.112 & $42.2(5.0)$ & $53.6(5.4)$ & 0.019 \\
\hline NSAIDs* $(\%)$ & $101(10.6)$ & $307(27.3)$ & 0.438 & $160.4(19.1)$ & $206.3(20.9)$ & 0.046 \\
\hline *Glucocorticoids (\%) & $16(1.7)$ & $17(1.5)$ & 0.013 & $35.6(4.2)$ & $16.3(1.7)$ & 0.153 \\
\hline
\end{tabular}

${ }^{*}$ Number of patients prescribed with drugs for $>50 \%$ of the interval period.

BASDAI, Bath Ankylosing Spondylitis Disease Activity Index; CDMARDs, conventional disease-modifying anti-rheumatic drugs; CRP, C-reactive protein; ESR, erythrocyte sedimentation rate; IPTW, inverse probability of treatment weighting; mSASSS, modified Stoke Ankylosing Spondylitis Spinal Score; NSAIDs, non-steroidal anti-inflammatory drugs mSASSS change per year with and without TNFi therapy; SMD, standardised mean difference; TNFis, tumour necrosis factor inhibitors. 
Table 5 The adjusted mSASSS changes per year when treated with or without TNFi from each statistical model

\begin{tabular}{lll}
\hline \multirow{2}{*}{ Model } & \multicolumn{2}{l}{ TNFi } \\
\cline { 2 - 3 } & On & Off \\
\hline Multivariable model & 0.914 & 0.970 \\
Marginal structural model & 0.848 & 0.960 \\
\hline
\end{tabular}

TNFi, tumour necrosis factor inhibitor.

$-0.036, \mathrm{p}=0.004$ with sandwich estimator and $95 \% \mathrm{CI}-0.168$ to $-0.056, \mathrm{p}<0.001$ with bootstrap).

We estimated the mSASSS change per year from the mSASSS change rate in the multivariable regression and the MSM with IPTW model (table 5). Adjusting other covariates, the mSASSS change per year was 0.914 and 0.848 in the multivariable regression and MSM models, respectively, in 'on the TNFi' interval, whereas in 'off the TNFi' intervals, these were 0.970 and 0.960 , respectively. The mSASSS change per year showed a greater decrease in the MSM with IPTW model than in the multivariable regression model.

\section{DISCUSSION}

We used 2364 intervals of 338 patients from the longitudinal data to determine the effects of TNFis on radiographic progression, focusing on the TNFi treatment period. Compared with other treatments, TNFi therapy slowed the radiographic progression, suggesting that TNFi therapy may play an important role in radiographic progression.

In previous randomised controlled trials with a short period of 2 years, TNFi therapy did not inhibit radiographic progression. ${ }^{11-13}$ Recently, long-term or early treatment with TNFi may influence radiographic progression. ${ }^{35}$ Nevertheless, obtaining enough data with sufficient duration to identify radiographic progression and avoiding selection bias in examining drug effects are difficult. To overcome these problems, we used real-world data derived from EMRs of patients with advanced statistical methods, such as MSM with IPTW with comparison between drug intervals, not between patient groups.

AS treatment usually begins with NSAIDs or cDMARDs. Despite such treatment, when the disease activity becomes severe, biologics such as TNFis are started. Radiological progression would be accelerated when the disease activity and inflammatory status worsen. ${ }^{1425}$ Therefore, patients with an indication for TNFi therapy are more likely to experience severe radiographic damage in the future than those who maintain therapy with NSAIDs or DMARDs. Given that we usually use TNFis in high inflammatory status, we should consider that TNFi treatment directly affects radiographic progression (direct effect) and slows radiographic progression by lowering the high inflammatory status (indirect effect) in study design for drug effect on radiographic change. However, regression methods that evaluate the effects of TNFis by fixing other covariates, such as ESR and CRP, cannot account for indirect effects.

Ramiro et al investigated radiographic progression in AS using 12-year data from the Outcome in Ankylosing Spondylitis International Study. ${ }^{6}$ They suggested that the radiographic damage rate was 0.98 mSASSS units per year. However, patients exposed to TNFis showed a higher progression rate than patients who were not exposed because of 'confounding by indication'. In a recent study, Park et al showed that early TNFi treatment can reduce radiographic progression by inflammation control. ${ }^{15}$ They enrolled a TNFi treatment group from one single-centre cohort and a conventional NSAID treatment group from another single cohort to analyse the effect of TNFi on radiographic progression. However, as mentioned in their limitation, they also have a problem with 'confounding by indication', as patients had significantly different baseline characteristics between periods exposed and not exposed to TNFi.

To avoid this bias, we defined intervals based on the period of treatment with or without TNFi therapy among patients who have been prescribed TNFi. It is expected that this method can reasonably explain the drug effects in contrast to recruiting of patients according to the inclusion criteria and dividing them into experimental and control groups. From these data, the rate of radiographic progression was identified using a multivariable regression model. Additionally, we used MSM with IPTW considering both direct and indirect effects of TNFi therapy. In both models, TNFi treatment significantly delayed radiographic progression compared with treatment without TNFi. Interestingly, radiographic progression of 'on the TNFi' interval was lower using MSM with IPTW than using multivariable regression. We suggested that this difference was from the indirect effect of TNFi through inflammation on radiographic progression and the confounding effect of time-varying covariates. Considering both effects of TNFis, we demonstrated not only the association but also the causal effect of TNFis on radiographic progression using MSM with IPTW. In the future, these statistical methods will be useful in evaluating the long-term effects of drug use on chronic rheumatic diseases using multi- EMR data.

We revealed some possible factors related to radiographic progression. The radiographic progression was not severe in women than in men, as reported in previous studies. ${ }^{26} 27$ However, unlike previous studies, ${ }^{28} 29$ eye involvement was associated with severe radiographic progression. We suggested that the inflammatory burden accelerating radiographic progression or association with HLA-B27 may also have affected uveitis development. ${ }^{30}$ There was a significant negative relationship between cDMARDs and mSASSS change rate. Although concomitant drugs were controlled for in the MSM with IPTW, the causal effect of concomitant drugs cannot be estimated. A separate study is needed to investigate the causal effect of cDMARDs on radiographic progression.

Among the parameters, BASDAI at the start of the interval was negatively associated with mSASSS change rate in the multivariable regression model, although positive correlations between ESR and mSASSS change rate were found. Several intervals with high BASDAI were included as the 'on the TNFi' intervals. Therefore, the results indicated that high BASDAI was associated with a decrease in mSASSS change rate in the multivariable regression model, which may be related to multicollinearity. Therefore, these issues made it clear that MSM with IPTW was the accurate solution to address the problem in our study.

This study has several limitations. First, although smoking has been known to have a significant effect of radiographic progression, it was not included in the covariates, because there were too many missing values for smoking status. Second, patients who had never been exposed to TNFi were not included. Thus, the mSASSS change rate in these patients may differ from that obtained in the 'off the TNFi' interval. Third, we imputed the mSASSS data with interpolation, because mSASSS tends to increase very slowly with radiological progression in the real world. However, the imputed values, such as those for inflammatory markers or disease activity, may differ from the actual values owing to the large variability during the observation period.

The rate of radiographic progression was different between periods in which patients were exposed and not exposed to TNFi. Our study provided strong evidence that TNFi therapy 
slows the radiographic progression by overcoming the limitations of previous studies. Treatment using TNFis is among the modifiable factors for radiographic progression in patients with AS.

\section{Author affiliations}

'Department of Internal Medicine, Inje University Seoul Paik Hospital, Inje University College of Medicine, Seoul, Republic of Korea

${ }^{2}$ Department of Biomedical Informatics, Asan Medical Center, Seoul, Republic of Korea

${ }^{3}$ Department of Clinical Epidemiology and Biostatistics, Asan Medical Center, University of Ulsan College of Medicine, Seoul, Republic of Korea

${ }^{4}$ Department of Rheumatology, Hanyang University Hospital for Rheumatic Diseases, Seoul, Republic of Korea

${ }^{5}$ Department of Rheumatology, Department of Internal Medicine, Korea University Guro Hospital, Seoul, Republic of Korea

${ }^{6}$ Department of Radiology, Hanyang University Hospital for Rheumatic Diseases, Seoul, Republic of Korea

Acknowledgements The authors would like to thank all members of Hanyang University Hospital for Rheumatic Diseases. Furthermore, the authors would also like to thank the nurses who have helped in data collection.

Contributors DrTHK had full access to all the data in the study and takes responsibility for the integrity of the data, study supervision and accuracy of the data analysis. All authors participated in the interpretation of data, drafting, revision and approval of the manuscript. BSK, JSO, SYP and THK contributed to the study conception and/or design. BSK, JSO and SYP help in the statistical analysis. JHS, GYA SL, KBJ and THK provided administrative, technical or material support.

Funding The authors have not declared a specific grant for this research from any funding agency in the public, commercial or not-for-profit sectors.

Competing interests None declared.

Patient and public involvement Requirement for informed consent was waived because this study was a retrospective review of electronic medical records.

Patient consent for publication Not required.

Ethics approval The study design was approved by the appropriate ethics review board.

Provenance and peer review Not commissioned; externally peer reviewed.

Data availability statement Study protocol and statistical code: Available on request from Dr Park (biostat81@amc.seoul.kr). Data set: Available on request and signature of a confidentiality agreement from Prof Kim (thkim@hanyang.ac.kr)

ORCID iD

Bon San Koo http://orcid.org/0000-0002-4212-2634

\section{REFERENCES}

1 Creemers MCW, Franssen MJAM, van't Hof MA, et al. Assessment of outcome in ankylosing spondylitis: an extended radiographic scoring system. Ann Rheum Dis 2005;64:127-9.

2 Poddubnyy D, Haibel H, Listing J, et al. Baseline radiographic damage, elevated acute-phase reactant levels, and cigarette smoking status predict spinal radiographic progression in early axial spondylarthritis. Arthritis Rheum 2012;64:1388-98.

3 Haroon N, Inman RD, Learch TJ, et al. The impact of tumor necrosis factor $\alpha$ inhibitors on radiographic progression in ankylosing spondylitis. Arthritis Rheum 2013:65:2645-54.

4 van der Heijde D, Braun J, Deodhar A, et al. Modified stoke ankylosing spondylitis spinal score as an outcome measure to assess the impact of treatment on structural progression in ankylosing spondylitis. Rheumatology 2019;58:388-400.

5 Baraliakos X, Haibel H, Listing J, et al. Continuous long-term anti-TNF therapy does not lead to an increase in the rate of new bone formation over 8 years in patients with ankylosing spondylitis. Ann Rheum Dis 2014;73:710-5.

6 Ramiro S, Stolwijk C, van Tubergen A, et al. Evolution of radiographic damage in ankylosing spondylitis: a 12 year prospective follow-up of the OASIS study. Ann Rheum Dis 2015:74:52-9.

7 Ramiro S, van der Heijde D, van Tubergen A, et al. Higher disease activity leads to more structural damage in the spine in ankylosing spondylitis: 12-year longitudinal data from the OASIS cohort. Ann Rheum Dis 2014;73:1455-61.
8 van der Heijde D, Ramiro S, Landewé R, et al. 2016 update of the ASAS-EULAR management recommendations for axial spondyloarthritis. Ann Rheum Dis 2017;76:978-91.

9 Ward MM, Deodhar A, Gensler LS, et al. 2019 update of the American College of Rheumatology/Spondylitis association of America/Spondyloarthritis research and treatment network recommendations for the treatment of ankylosing spondylitis and Nonradiographic axial spondyloarthritis. Arthritis Rheumatol 2019.

10 Baraliakos X, Listing J, Rudwaleit M, et al. Radiographic progression in patients with ankylosing spondylitis after 2 years of treatment with the tumour necrosis factor alpha antibody infliximab. Ann Rheum Dis 2005:64:1462-6.

11 van der Heijde D, Landewé R, Baraliakos $X$, et al. Radiographic findings following two years of infliximab therapy in patients with ankylosing spondylitis. Arthritis Rheum 2008;58:3063-70

12 van der Heijde $D$, Landewé $R$, Einstein $S$, et al. Radiographic progression of ankylosing spondylitis after up to two years of treatment with etanercept. Arthritis Rheum 2008:58:1324-31.

13 van der Heijde D, Salonen D, Weissman BN, et al. Assessment of radiographic progression in the spines of patients with ankylosing spondylitis treated with adalimumab for up to 2 years. Arthritis Res Ther 2009;11:R127.

14 Molnar C, Scherer A, Baraliakos X, et al. Tnf blockers inhibit spinal radiographic progression in ankylosing spondylitis by reducing disease activity: results from the Swiss clinical quality management cohort. Ann Rheum Dis 2018;77:63-9.

15 Park JW, Kim MJ, Lee JS, et al. Impact of tumor necrosis factor inhibitor versus nonsteroidal antiinflammatory drug treatment on radiographic progression in early ankylosing spondylitis: its relationship to inflammation control during treatment. Arthritis Rheumatol 2019;71:82-90.

16 Haroon N, Maksymowych WP, Rahman P, et al. Radiographic severity of ankylosing spondylitis is associated with polymorphism of the large multifunctional peptidase 2 gene in the spondyloarthritis research Consortium of Canada cohort. Arthritis Rheum 2012;64:1119-26.

17 Maas F, Arends S, Brouwer E, et al. Reduction in spinal radiographic progression in ankylosing spondylitis patients receiving prolonged treatment with tumor necrosis factor inhibitors. Arthritis Care Res 2017:69:1011-9.

18 Corrigan-Curay J, Sacks L, Woodcock J. Real-World evidence and real-world data for evaluating drug safety and effectiveness. JAMA 2018;320:867-8.

19 Misra DP, Agarwal V. Real-World evidence in rheumatic diseases: relevance and lessons learnt. Rheumatol Int 2019:39:403-16.

20 van der Linden S, Valkenburg HA, Cats A. Evaluation of diagnostic criteria for ankylosing spondylitis. A proposal for modification of the new York criteria. Arthritis Rheum 1984;27:361-8

21 Garrett S, Jenkinson T, Kennedy LG, et al. A new approach to defining disease status in ankylosing spondylitis: the Bath ankylosing spondylitis disease activity index. J Rheumatol 1994:21:2286-91.

22 Hernán MA, Brumback B, Robins JM. Marginal structural models to estimate the causal effect of zidovudine on the survival of HIV-positive men. Epidemiology 2000;11:561-70

23 Robins JM, Hernán MA, Brumback B. Marginal structural models and causal inference in epidemiology. Epidemiology 2000;11:550-60.

24 Hernan MA, Robins JM. Causal inference: what if. Boca Raton: Chapman \& Hall/CRC 2020.

25 Poddubnyy D, Protopopov M, Haibel H, et al. High disease activity according to the ankylosing spondylitis disease activity score is associated with accelerated radiographic spinal progression in patients with early axial spondyloarthritis: results from the German spondyloarthritis inception cohort. Ann Rheum Dis 2016:75:2114-8.

26 Lee W, Reveille JD, Davis JC, et al. Are there gender differences in severity of ankylosing spondylitis? results from the psoas cohort. Ann Rheum Dis 2007:66:633-8

27 van Tubergen A, Ramiro S, van der Heijde D, et al. Development of new syndesmophytes and bridges in ankylosing spondylitis and their predictors: a longitudinal study. Ann Rheum Dis 2012;71:518-23.

28 Chen $\mathrm{H}-\mathrm{A}$, Chen $\mathrm{C}-\mathrm{H}$, Liao $\mathrm{H}-\mathrm{T}$, et al. Factors associated with radiographic spinal involvement and hip involvement in ankylosing spondylitis. Semin Arthritis Rheum 2011:40:552-8

29 Essers I, Ramiro S, Stolwijk C, et al. Do extra-articular manifestations influence outcome in ankylosing spondylitis? 12-year results from OASIS. Clin Exp Rheumatol 2016;34:214-21.

30 Sun L, Wu R, Xue Q, et al. Risk factors of uveitis in ankylosing spondylitis: an observational study. Medicine 2016;95:e4233. 